\title{
Effects of environment on the properties of cluster galaxies via ram pressure stripping
}

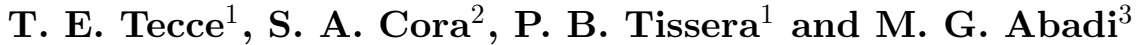 \\ ${ }^{1}$ Instituto de Astronomía y Física del Espacio, CONICET-UBA, CC 67 Suc. 28, C1428ZAA \\ Buenos Aires, Argentina. \\ ${ }^{2}$ Facultad de Ciencias Astronómicas y Geofísicas, UNLP and Instituto de Astrofísica de La \\ Plata, CONICET, Paseo del Bosque S/N, B1900FWA La Plata, Argentina. \\ ${ }^{3}$ Observatorio Astronómico, UNC, Laprida 854, X5000BGR Córdoba, Argentina. \\ email: tomas@iafe.uba.ar
}

\begin{abstract}
We study the effect of ram pressure stripping (RPS) on the colours, cold gas content and star formation of galaxies in clusters, using a combination of $N$-Body/SPH simulations of galaxy clusters and a semi-analytic model of galaxy formation that includes the effect of RPS.
\end{abstract}

Keywords. Galaxies: clusters: general, galaxies: evolution, intergalactic medium

In the local universe, galaxy clusters have larger fractions of red, early-type galaxies than the field (Baldry et al. 2006) and star formation is suppressed in denser environments (Kauffmann et al. 2004). This could be caused by removal of cold gas from galaxies via ram pressure stripping (RPS) by the intracluster medium (ICM) (Gunn \& Gott 1972).

We have studied the influence of RPS on galaxy properties using a hybrid model that combines non-radiative $N$-Body/SPH cosmological simulations of clusters with masses in the range $10^{14}-10^{15} h^{-1} \mathrm{M}_{\odot}$ (Dolag et al. 2005) and a semi-analytic model of galaxy formation (Lagos et al. 2008) in which we implement the RPS process. We use the information provided by the gas particles in the simulations to obtain the properties of the ICM and the velocities of galaxies relative to it. This results in a more self-consistent method than previous works, which relied on analytical approximations.

We have run two sets of models, with and without RPS, with all the parameters involved in other physical processes remaining unchanged. Our results show that RPS is more important in the more massive haloes, but becomes significant only at $z \lesssim 0.5$. In the RPS model, star formation activity is strongly suppressed in dwarf galaxies $\left(\mathrm{M}_{*}<10^{10} h^{-1} \mathrm{M}_{\odot}\right)$, which lose most of their ISM and become red and passive on a shorter timescale. RPS could then be responsible for the loss of gas in cluster dE galaxies (Boselli et al. 2008). Mean colours of the red and blue sequences do not change significantly, but the number of faint red galaxies increases in the RPS model for $z<1$. The fraction of gas poor galaxies in regions around clusters increase from $\sim 5 \%$ to $\sim 20 \%$, indicating that RPS acts to some degree in group environments prior to cluster infall.

\section{References}

Baldry, I. K. et al. 2006, MNRAS 373, 469

Boselli, A. et al. 2008, ApJ 674, 742

Dolag, K. et al. 2005, MNRAS 364, 753

Gunn, J. E. \& Gott, J. R. 1972, ApJ 176, 1

Kauffmann, G. et al. 2004, MNRAS 353, 713

Lagos, C. D. P., Cora, S. A., \& Padilla, N. D. 2008, MNRAS 388, 587 